
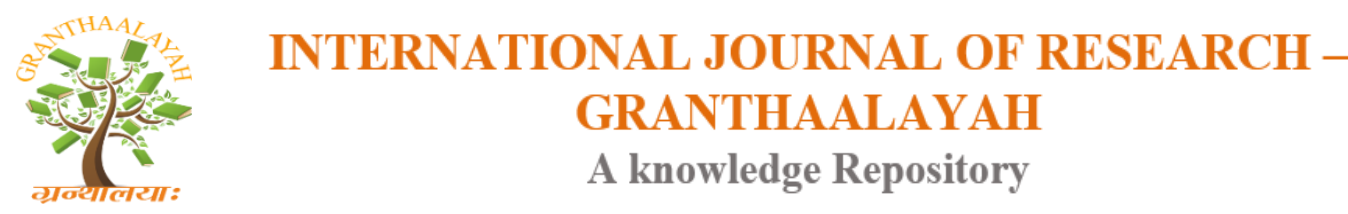

DOI: 10.29121/granthaalayah.v6.i3.2018.1496

Science

\title{
ACTION THRESHOLD LEVELS OF HELICOVERPA ARMIGERA (HBN.) ON COTTON AT DIFFERENT STAGES OF PLANT GROWTH
}

\author{
Amir Abdullahi Yousif Malik ${ }^{* 1}$, Elamin Mohamed Elamin ${ }^{1}$ \\ *1 Agricultural Research Corporation (ARC), Wad Medani, Sudan
}

\begin{abstract}
The African bollworm, Helicoverpa armigera (Hbn.) (Lepidoptera: Noctuidae), is the most important cotton pest in all the cotton growing areas of the Sudan and most of the protection measures are directed for its management. The previous action threshold level for chemical control interference was increased in 1993 from 10 larvae and/or eggs /100 plants to 30 eggs or 10 larvae/100 plants and no spray to be conducted before flowering advances. This level was being subject to revision since 1999. Studies conducted at the Gezira Research Farm during 2001/2002, 2002/2003, 2003/2004, 2004/2005 and 2012/2013 were carried out on this regard at growth stages of cotton plants, i.e. Pre-flowering, flowering and bolls formation stages and the consequence on yield. Barac. (67) B cultivar, Gossypium hirsutum, was used. The action threshold levels were 10, 15, 20 eggs and/or larvae/100 plants and 30 eggs or 10 larvae/100 plants, in addition to untreated and Helicoverpa infestation free as control treatments. The results of damage on fruiting bodies (squares, flowers and bolls) and seed cotton yield showed that there were no significant differences between the tested action threshold levels in the pre-flowering and the flowering stages of cotton growth as compared to the control treatments. From the yields and number of sprays in the treatments it can be concluded that the already recommended action threshold 30 eggs or 10 larvae/100 plants is still be applied when flowering advances and bolls formed. Moreover, the standing action threshold seems lower than the actual level. Therefore, it is suggested to investigate higher action threshold than that in current use.
\end{abstract}

Keywords: Action Threshold Level; Helicoverpa Armigera; Cotton; Sudan.

Cite This Article: Amir Abdullahi Yousif Malik, and Elamin Mohamed Elamin. (2018). "ACTION THRESHOLD LEVELS OF HELICOVERPA ARMIGERA (HBN.) ON COTTON AT DIFFERENT STAGES OF PLANT GROWTH." International Journal of Research Granthaalayah, 6(3), 39-48. https://doi.org/10.29121/granthaalayah.v6.i3.2018.1496.

\section{Introduction}

The African bollworm (ABW), Helicoverpa armigera (Hbn.) (Lepidoptera: Noctuidae), is a polyphagus insect pest of a wide range of distribution. It is a pest in almost all cotton regions of the Sudan. Cotton is attacked by the ABW late September/early October and infestation continues to about February and three of four generations complete their development on cotton 
(Balla, 1979). At present, most of the protection operations are directed for its management. The extent of damage to the host plant is determined by the feeding habits of the larvae. On cotton, the newly hatched caterpillar starts feeding on the youngest buds, and, as it grows older, moves to the larger buds, flowers and in the last stages the larva attacks also the bolls (Schmutterer, 1969).

Plant Protection costs had steadily increased since the seventieth of the previous century, whereas average yields remained almost unchanged. The research conducted by the Integrated Pest Management Project (ARC-FAO, 1979-1997) showed that good cotton yields could be obtained with reduced use of insecticides and elevated the action threshold. The term action threshold is of particular importance, as it can be defined as "the control level that is needed to prevent a population from reaching the economic threshold", while the economic threshold can be defined as: "the level of infestation of (an) insect (s) at which the losses due to infestation exceed the costs for control and levels are usually expressed in terms of population densities" (Barendregt and van Lenteren. 1992). The Economic Threshold Level (ETL) (action threshold level) for the four major cotton pests (Whitefly, Jassid, Aphid and the African bollworm) were increased. The current action threshold level for the ABW is 30 eggs or 10 larvae / 100 plants and no insecticides spraying should commence before flowering advances (Abdelrahman et al., 1991 and Stam et al., 1994). This was recommended in 1993 by the National Pests and Diseases Committee and in Sept., 1999 one of the main recommendations of the Workshop on the ABW held at ARC was to revise this level.

The effects on yield were found to depend not only on the number of larvae present, but also on their size, the timing of the infestation and the amount of damage done previously (Wallach, 1980). Abdelrahman (1995) showed that all the commercial cotton varieties can compensate the damage caused by $\mathrm{H}$. armigera up to four weeks after the initiation of flowering. Eveleens and Abdelrahman (1980) mentioned that the first insecticide application can be delayed. As a result, during 1985-1992 a series of trails to raise the ETLs were conducted in different localities (Stam et al., 1994). Beside the ETL; 30 eggs or 10 larvae/ 100 plants which raised in 1993, also the IPM Project experimented the threshold 20 eggs and/or larvae per 100 plants in Gezira Scheme, Amara Kassir Block in an area of 5000 fed and gave good results. Thus, the impact of insect attack on the performance of the crop depends on the stage in which the host plant is attacked (Barendregt and van Lenteren, 1992). However, the effects on yield, depending on an effective action threshold level at different stages of cotton growth, have not been sufficiently evaluated in the Sudan. Therefore, this experiment was designed to study the effects of H. armigera on yield of cotton with reference to action threshold levels at different stages of cotton growth.

\section{Materials and Methods}

This experiment was conducted in Gezira Research Farm in seasons; 2001/2002, 2002/2003, 2003/2004, 2004/2005 and 2012/2013. The different stages of cotton growth were namely; the pre-flowering stage $(\mathrm{X})\{45-75$ days after emergence $\}$, the flowering stage (Y) $\{75-105$ days after emergence $\}$ and the boll formation stage $(\mathrm{Z})\{105$ days to early opening of bolls $\}$. Four action threshold levels were proposed at the three stages of cotton growth in addition to infestation throughout (U) and Helicoverpa infestation free (T) as control treatments. These 
levels were: 10 eggs and/or larvae/100 plants (A), 15 eggs and/or larvae/100 plants (B), 20 eggs and/or larvae/100 plants (C), 30 eggs or 10 larvae/100 plants (D). So, 14 treatments were used. The experimental design used was randomized complete block design (RCBD) with 4 replications. The experimental area was $6720 \mathrm{~m} 2$ (1.6 feddan), divided into 56 sub-plots, the area of each sub-plot was 10X12 m. Acala, Barac. (67) B cotton variety was sown on July 29, 2001, July 27, 2002, July 16, 2003, July 15, 2004 and July 25, 2012. The cotton seeds were dressed with Gaucho WS 70 + Raxil $2 \%$ WS at the rate of $7 \mathrm{~g} / \mathrm{kg}$ and $2 \mathrm{~g} / \mathrm{kg}$ of cotton seeds, respectively.

The cultural practices were carried out as recommended by the ARC, but in season 2002/2003 the irrigation was irregular during October because of the water shortage in the minor canal and also in season 2004/2005 the 1st weeding was delayed as a result of the continuous rains in August; also the irrigation during mid September to mid October fluctuated due to the water shortage. Picking was carried out by hand and the first picking was started on December 31, 2001, December 16, 2002, January 18, 2003, 1st December, 2004 and 1st December, 2012. While, the second picking was completed on March 4, 2002, March 14, 2003, February 28, 2004, January 30, 2005 and January 30, 2013 for the five seasons, respectively.

All the treatments in this experiment except " $\mathrm{U}$ " and "T" were divided according to the phase of cotton growth into three stages:

1) Pre-flowering stage, $45-75$ days after emergence.

2) Flowering stage, 75-105 days after emergence.

3) Boll formation stage, 105 days after emergence to early opening of bolls.

These stages were equivalent to 3,4 and 5 of the segmented growth stages of cotton set by Walker (1971).

For treatment "T" and the stage(s) of cotton growth decided to be free of H. armigera infestation, the deposited eggs were removed manually every 48 hours.

Different sampling procedures were carried out for $\mathrm{H}$. armigera beside the natural enemies. Concerning H. armigera, the number of damaged and undamaged squares, flowers and bolls per 20 plants per sub-plot were weekly recorded. The percentage damage was calculated from the total number of fruiting bodies recorded. Eggs and larvae in randomly sampled 20 plants/subplot were weekly counted. The mean numbers of the important natural enemies (adult predators) at different sampling dates were recorded (Appendix 1).

The insecticides; Avaunt 150 SC (a selective insecticide recommended for the control of the $\mathrm{ABW}$ ) at 0.105 litre/fed (15.75 g a.i.) and Tracer $240 \mathrm{SC}$ (spinosad) at 0.104 litre/fed (25 g a.i.) were used. The insecticides were applied by a knapsack sprayer for spraying the treatments that reached the suggested action threshold levels.

The yield data and the damaged bolls were subjected to analysis of variance (ANOVA) and Duncan's Multiple Range Test whenever applicable. Meteorological data on temperature, rainfall and relative Humidity (R.H. \%) during the five seasons were obtained from the Agrometeorological Services Department at Wad Medani (Table 1). 
Appendix 1: Number of natural enemies (adult predators) encountered in the experimental plots, G.R.F, season; 2001/2002, 2002/2003, 2003/2004, 2004/2005 and 2012/2013

\begin{tabular}{|c|c|c|c|c|c|c|c|c|c|c|c|c|c|c|c|c|c|c|c|c|c|c|c|c|c|}
\hline \multirow[t]{2}{*}{\begin{tabular}{|l} 
Tre \\
at.
\end{tabular}} & \multicolumn{5}{|c|}{$\begin{array}{c}\text { Chrysoperla/10 } \\
0 \text { leaves }\end{array}$} & \multicolumn{5}{|c|}{$\begin{array}{c}\text { Coccinellids/100 } \\
\text { leaves }\end{array}$} & \multicolumn{5}{|c|}{$\begin{array}{c}\text { Syrphid/100 } \\
\text { leaves }\end{array}$} & \multicolumn{5}{|c|}{$\begin{array}{c}\text { Campylomma/100 } \\
\text { leaves }\end{array}$} & \multicolumn{5}{|c|}{$\begin{array}{c}\text { Spiders/100 } \\
\text { leaves }\end{array}$} \\
\hline & 1 & 2 & 3 & 4 & 5 & 1 & 2 & 3 & 4 & 5 & 1 & 2 & 3 & 4 & 5 & 1 & 2 & 3 & 4 & 5 & 1 & 2 & 3 & 4 & 5 \\
\hline XA & 1 & 0 & 1 & 0 & 2 & 2. & 0 & 0 & 0 & 1 & 0 . & 0. & 0 & 0 & 0 & 4. & 1 & 7 & 0. & 4 & 1. & 1 & 2 & 0 & 7. \\
\hline XB & . & & 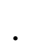 & 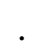 & & 3 & & 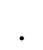 & & & 0 & 0 & & & & 2 & & & 9 & 2. & 5 & & . & & 9 \\
\hline $\mathrm{XC}$ & 6 & 8 & 3 & 1 & 5 & 5 & 4 & 4 & 3 & 8 & 3 & 0 & 0 & 0 & 0 & 5 & 2 & 4 & 1. & 4 & 0 & 4 & 6 & 6 & 8. \\
\hline XD & 2 & 0 & 2 & 0 & 2 & 2. & 0 & 0 & 0 & 1 & 0 . & 0 & 0 & 0 & 0 & 3. & 1 & 6 & 0 & 3 & 1. & 1 & 3 & 0 & 1 \\
\hline YA & . & & 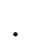 & . & & 5 & & . & & & 0 & 0. & 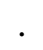 & & & 6 & & & 0. & 9. & 5 & & . & & 9. \\
\hline YB & 3 & 8 & 0 & 2 & 5 & 3 & 4 & 5 & 1 & 4 & 3 & 0 & 0 & 0 & 0 & 4 & 6 & 4 & 4 & 3 & 0 & 6 & 0 & 7 & 3 \\
\hline YC & 1 & 1 & 2 & 0 & 4 & 2. & 0 & 0 & 0 & 0 & 0 . & 1 & 0 & 0 & 0 & 3. & 1 & 7 & 1. & 2 & 2. & 1 & 3 & 0 & 7. \\
\hline YD & . & & 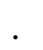 & . & & 1 & & 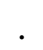 & & & 0 & 0. & . & & & 2 & . & & 4 & 3. & 8 & & . & & 1 \\
\hline $\mathrm{ZA}$ & 8 & 2 & 3 & 1 & 3 & 7 & 2 & 4 & 1 & 7 & 0 . & 0 & 0 & 0 & 0 & 5 & 2 & 4 & 1. & 6 & 9 & 0 & 0 & 4 & 1 \\
\hline $\mathrm{ZB}$ & 1 & 0 & 1 & 0 & 1 & 2. & 0 & 0 & 0 & 1 & 0 & 0. & 0 & 0 & 0 & 2. & 1 & 8 & 2 & 3 & 1. & 1 & 2 & 0 & 0 . \\
\hline $\mathrm{ZC}$ & . & . & . & . & . & 4 & 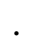 & . & 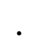 & & 3 & 0 & . & & . & 6 & 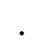 & & 1. & 7. & 9 & & . & & 0 \\
\hline ZD & 4 & 8 & 4 & 2 & 8 & 6 & 8 & 2 & 2 & 1 & 0. & 0 & 3 & 0 & 0 & 0 & 4 & 2 & 4 & 5 & 6 & 4 & 7 & 7 & 8. \\
\hline $\mathrm{U}$ & 1 & 1 & 2 & 0 & 1 & 2. & 0 & 0 & 0 & 2 & 0 & 0 & 0 & 0 & 0 & 4. & 1 & 9 & 1. & 3 & 1. & 1 & 3 & 0 & 9 \\
\hline $\mathrm{T}$ & 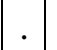 & . & . & $\cdot$ & . & 7 & $\cdot$ & . & $\cdot$ & & 3 & 0. & . & & . & 5 & . & & 2 & 6. & 7 & . & . & & 1 \\
\hline & 8 & 2 & 7 & 2 & 1 & 8 & 4 & 1 & 1 & 1 & 0. & 0 & 0 & 0 & 0 & 0 & 0 & 6 & 0. & 4 & 1 & 0 & 4 & 6 & 1. \\
\hline & 1 & 1 & 1 & 0 & 2 & 2. & 0 & 0 & 0 & 0 & 0 & 0. & 0 & 0 & 0 & 3. & 1 & 6 & 7 & 3 & 1. & 1 & 2 & 0 & 4 \\
\hline & . & 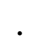 & . & . & . & 5 & 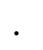 & 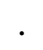 & 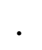 & 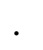 & 0. & 0 & . & 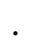 & . & 1 & . & & 1. & 6. & 5 & - & . & - & 5. \\
\hline & 4 & 2 & 8 & 2 & 9 & 7 & 2 & 1 & 1 & 4 & 0 & 1 & 1 & 1 & 0 & 0 & 2 & 3 & 4 & 1 & 3 & 2 & 6 & 8 & 0 \\
\hline & 1 & 0 & 1 & 0 & 2 & 2. & 0 & 0 & 0 & 1 & 3 & 0. & 0 & 0 & 0 & 2. & 2 & 6 & 1. & 3 & 1. & 2 & 2 & 0 & 7. \\
\hline & . & • & . & . & . & 2 & $\cdot$ & 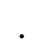 & $\cdot$ & & 0 . & 0 & 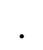 & & & 7 & . & & 5 & 4. & 8 & & . & & 0 \\
\hline & 8 & 8 & 5 & 5 & 9 & 5 & 2 & 2 & 2 & 4 & 0 & 0. & 0 & 0 & 0 & 1 & 0 & 6 & 1. & 9 & 9 & 2 & 9 & 4 & 6. \\
\hline & 2 & 0 & 1 & 0 & 1 & 2. & 0 & 0 & 0 & 1 & 0. & 2 & 0 & 0 & 0 & 5. & 1 & 7 & 2 & 4 & 1. & 1 & 2 & 0 & 8 \\
\hline & . & . & . & $r$ & . & 6 & 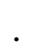 & . & . & & 0 & 0. & 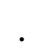 & & . & 2 & . & & 1. & 1. & 6 & & . & & 9. \\
\hline & 1 & 8 & 9 & 4 & 1 & 7 & 2 & 1 & 2 & 1 & 3 & 0 & 0 & 0 & 0 & 5 & 8 & 0 & 2 & 4 & 0 & 6 & 9 & 7 & 6 \\
\hline & 2 & 1 & 2 & 0 & 3 & 2. & 0 & 0 & 0 & 1 & 0. & 1 & 0 & 0 & 0 & 3. & 1 & 8 & 1 & 3 & 1. & 1 & 3 & 0 & 6. \\
\hline & . & . & $\cdot$ & . & ${ }^{\circ}$ & 3 & . & . & . & & 0 & 0. & . & 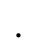 & & 0 & 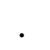 & & 1. & 4. & 6 & & . & & 1 \\
\hline & 0 & 0 & 7 & 0 & 9 & 2 & 4 & 1 & 2 & 1 & 0. & 0 & 1 & 0 & 0 & 7 & 6 & 5 & 3 & 4 & 4 & 0 & 0 & 6 & 1 \\
\hline & 2 & 0 & 1 & 0 & 3 & 3. & 0 & 0 & 0 & 0 & 0 & 0. & 0 & 0 & 0 & 4. & 2 & 7 & 1. & 3 & 1. & 1 & 3 & 0 & 1. \\
\hline & . & $\cdot$ & . & + & 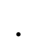 & 1 & & . & $\cdot$ & & 0. & 0 & 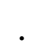 & & & 9 & 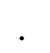 & & 1 & 0. & 3 & & . & & 1 \\
\hline & 1 & 8 & 6 & 0 & 2 & 7 & 6 & 4 & 1 & 4 & 0 & 0. & 0 & 0 & 0 & 6 & 0 & 3 & & 0 & 5 & 4 & 6 & 7 & 5. \\
\hline & 2 & 1 & 1 & 0 & 0 & 2. & 0 & 0 & 0 & 1 & 0. & 2 & 0 & 0 & 0 & 5. & 2 & 9 & & 3 & 1. & 2 & 2 & 0 & 7 \\
\hline & $0^{\circ}$ & ${ }^{\circ}$ & . & 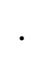 & 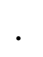 & 7 & & $\cdot$ & $\cdot$ & & 0 & & . & & & 4 & . & & & 0. & 7 & & 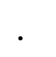 & & \\
\hline & 1 & 6 & 9 & 2 & 4 & 1 & 6 & 2 & 0 & 8 & 0. & & 0 & 0 & 0 & 2 & 0 & 4 & & 8 & 8 & 0 & 8 & 8 & \\
\hline & 1 & 0 & 2 & 0 & 5 & 2. & 0 & 0 & 0 & 1 & 0 & & 0 & 0 & 0 & 4. & 1 & 5 & & 3 & 1. & 1 & 3 & 0 & \\
\hline & . & 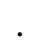 & 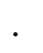 & $\cdot$ & & 6 & $\cdot$ & ${ }^{\circ}$ & $\cdot$ & & & & $\cdot$ & & & 2 & & & & 5. & 7 & & & & \\
\hline & 3 & 8 & 9 & 3 & 2 & 4 & 4 & 1 & 1 & 1 & & & 0 & 0 & 0 & 5 & 4 & 4 & & 7 & 1 & 0 & 1 & 7 & \\
\hline & 1 & 0 & 3 & 0 & 3 & 2. & 0 & 0 & 0 & 2 & & & 0 & 0 & 0 & 4. & 1 & 9 & & 3 & 1. & 1 & 3 & 0 & \\
\hline & . & $\cdot$ & 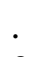 & $\cdot$ & ${ }^{\circ}$ & 7 & & 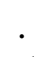 & $\cdot$ & & & & - & . & & 0 & . & 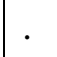 & & 5. & 7 & & . & & \\
\hline & 9 & 8 & 3 & 4 & 4 & 5 & 2 & 5 & 2 & 4 & & & 0 & 0 & 0 & 3 & 6 & 6 & & 4 & 5 & 6 & 1 & 8 & \\
\hline & 1 & 1 & 2 & 0 & 3 & 3. & 0 & 0 & 0 & 1 & & & 0 & 0 & 0 & 3. & 1 & 8 & & 2 & 1. & 1 & 3 & 0 & \\
\hline & ${ }^{\circ}$ & 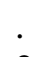 & 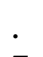 & 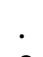 & $\cdot$ & 2 & 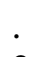 & 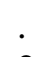 & $\cdot$ & & & & . & $\cdot$ & & 4 & . & & & 6. & 5 & & . & & \\
\hline & 9 & 2 & 7 & 2 & 6 & 8 & 2 & 2 & 0 & 4 & & & 0 & 0 & 0 & 6 & 6 & 7 & & 1 & 7 & 8 & 8 & 9 & \\
\hline
\end{tabular}


Table 1: The mean monthly maximum and mean minimum temperatures, relative humidity and total rainfall during the seasons; 2001/2002, 2002/2003, 2003/2004, 2004/2005 and 2012/2013

\begin{tabular}{|l|l|l|l|l|l|l|l|l|l|l|l|l|l|l|l|l|l|l|l|l|}
\hline $\begin{array}{l}\text { Mo } \\
\text { nth }\end{array}$ & \multicolumn{1}{|c|}{ Mean Max. Temp. ${ }^{\mathbf{0}}$ C } & \multicolumn{1}{|c|}{ Mean Min. Temp. ${ }^{\mathbf{C}}$} & \multicolumn{3}{|c|}{ Mean R.H.\% } & \multicolumn{3}{c|}{ Total R.F.(mm) } \\
\hline & 1 & 2 & 3 & 4 & 5 & 1 & 2 & 3 & 4 & 5 & 1 & 2 & 3 & 4 & 5 & 1 & 2 & 3 & 4 & 5 \\
\hline July & 36 & 38 & 35 & 38 & 35 & 23 & 24 & 23 & 23 & 23 & 7 & 6 & 7 & 6 & 6 & 13 & 53 & 10 & 19 & 4. \\
& .2 & .3 & .7 & .4 & .8 & .8 & .5 & .4 & .9 & .1 & 1 & 1 & 5 & 1 & 3 & 3.7 & .4 & 1.9 & .6 & 4 \\
\hline Aug & 34 & 35 & 33 & 36 & 34 & 22 & 22 & 22 & 23 & 22 & 8 & 7 & 8 & 7 & 6 & 33. & 84 & 15 & 99 & 89 \\
. & .1 & .9 & .4 & .3 & .0 & .7 & .8 & .5 & .4 & .1 & 2 & 6 & 3 & 3 & 9 & 3 & .3 & 2.1 & .9 & .1 \\
\hline Sept & 36 & 35 & 36 & 37 & 37 & 22 & 25 & 22 & 23 & 22 & 7 & 7 & 7 & 6 & 6 & 50. & 92 & 51. & 10 & 3. \\
. & .7 & .5 & .6 & .8 & .5 & .6 & .7 & .3 & .3 & .9 & 6 & 5 & 6 & 7 & 0 & 1 & .1 & 0 & .9 & 7 \\
\hline Oct. & 38 & 38 & 39 & 39 & 38 & 22 & 27 & 22 & 22 & 22 & 5 & 5 & 5 & 5 & 4 & 5.7 & 16 & 11. & 11 & 39 \\
& .9 & .9 & .1 & .3 & .8 & .0 & .9 & .9 & .6 & .1 & 9 & 4 & 6 & 0 & 8 & & .1 & 8 & .3 & .6 \\
\hline Nov & 37 & 38 & 38 & 38 & 37 & 18 & 20 & 20 & 20 & 18 & 4 & 4 & 4 & 4 & 4 & 0.4 & 0. & 0.0 & 0. & 0. \\
. & .7 & .1 & .1 & .1 & .1 & .8 & .1 & .2 & .1 & .7 & 5 & 3 & 5 & 0 & 0 & & 0 & & 0 & 0 \\
\hline Dec. & 35 & 33 & 35 & 33 & 35 & 17 & 14 & 15 & 16 & 15 & 4 & 3 & 4 & 4 & 3 & 0.0 & 0. & 0.0 & 0. & 0. \\
& .7 & .4 & .3 & .8 & .4 & .5 & .6 & .9 & .6 & .2 & 7 & 9 & 5 & 5 & 1 & & 0 & & 0 & 0 \\
\hline Jan. & 31 & 34 & 33 & 32 & 34 & 13 & 15 & 15 & 13 & 17 & 4 & 4 & 3 & 3 & 3 & 0.0 & 0. & 0.0 & 0. & 0. \\
& .5 & .8 & .9 & .3 & .9 & .2 & .5 & .6 & .9 & .3 & 3 & 1 & 9 & 7 & 3 & & 0 & & 0 & 0 \\
\hline Feb. & 36 & 36 & 34 & 38 & 37 & 17 & 17 & 16 & 20 & 20 & 3 & 2 & 3 & 4 & 2 & 0.0 & 0. & 0.0 & 0. & 0. \\
& .5 & .6 & .9 & .8 & .9 & .8 & .1 & .6 & .7 & .0 & 9 & 8 & 6 & 0 & 7 & & 0 & & 0 & 0 \\
\hline
\end{tabular}

Source: (Wad Medani Agro-meteorological Station).

Legend: $1=$ Season 2001/2002, 2= Season 2002/2003, 3= Season 2003/2004, 4= Season 2004/2005 \& 5= Season 2012/2013

\section{Results and Discussion}

Data on $\%$ damage differed according to the infestation level and the stage of the growth, damaged bolls in different treatments in the five seasons; 2001/2002, 2002/2003, 2003/2004, 2004/2005 and 2012/2013, showed significant differences between the control and all other treatments $(\mathrm{P}>0.05)$, as shown in Table 2 and 3.

Table 2: Mean number of damaged bolls, seasons; 2001/2002, 2002/2003, 2003/2004, 2004/2005 and 2012/2013

\begin{tabular}{|l|l|l|l|l|ll|ll|}
\hline Treat. & \multicolumn{7}{|c|}{ Mean number of damaged bolls } & \multicolumn{2}{|c|}{$\begin{array}{c}\text { Combined } \\
\text { analysis }\end{array}$} \\
\hline & 1 & 2 & 3 & 4 & 5 & & & \\
\hline $\mathrm{XA}$ & $1.024(0.05)$ & $1.114(0.25)$ & $1.000(0.0)$ & $1.000(0.0)$ & 1.021 & $(0.04)$ & 1.032 & $\mathrm{~A}$ \\
& $\mathrm{EF}$ & $\mathrm{AB}$ & $\mathrm{E}$ & $\mathrm{C}$ & $\mathrm{D}$ & & & \\
\hline $\mathrm{XB}$ & $1.000(0.0)$ & $1.024(0.05)$ & $1.000(0.0)$ & $1.035(0.08)$ & 1.006 & $(0.01)$ & 1.013 & $\mathrm{~A}$ \\
& $\mathrm{~F}$ & $\mathrm{~B}$ & $\mathrm{E}$ & $\mathrm{BC}$ & $\mathrm{D}$ & & & \\
\hline $\mathrm{XC}$ & $1.070(0.15)$ & $1.072(0.15)$ & $1.035(0.07)$ & $1.035(0.08)$ & 1.003 & $(0.01)$ & 1.043 & $\mathrm{~A}$ \\
& $\mathrm{DEF}$ & $\mathrm{AB}$ & $\mathrm{DE}$ & $\mathrm{BC}$ & $\mathrm{D}$ & & & \\
\hline $\mathrm{XD}$ & $1.048(0.10)$ & $1.253(0.60)$ & $1.035(0.07)$ & $1.000(0.0)$ & $1.002(0.003)$ & 1.068 & $\mathrm{~A}$ \\
& $\mathrm{DEF}$ & $\mathrm{A}$ & $\mathrm{DE}$ & $\mathrm{C}$ & $\mathrm{D}$ & & \\
\hline $\mathrm{YA}$ & $1.382(0.93) \mathrm{ABCD}$ & $1.081(0.18)$ & $1.126(0.27)$ & $1.146(0.33)$ & 1.172 & $(0.38)$ & 1.181 & $\mathrm{~A}$ \\
& & $\mathrm{AB}$ & $\mathrm{CDE}$ & $\mathrm{BC}$ & $\mathrm{B}$ & & & \\
\hline
\end{tabular}




\begin{tabular}{|c|c|c|c|c|c|c|}
\hline YB & $1.658(1.81) \mathrm{AB}$ & $\begin{array}{l}1.189(0.43) \\
\mathrm{AB}\end{array}$ & $\begin{array}{l}\text { 1.161(0.35) } \\
\text { BCDE }\end{array}$ & $\begin{array}{l}1.454(1.15) \\
\mathrm{A}\end{array}$ & $\begin{array}{l}1.165(0.36) \\
B\end{array}$ & $1.325 \mathrm{~A}$ \\
\hline YC & 1.514(1.31)ABC & $\begin{array}{l}1.059(0.12) \\
\mathrm{AB}\end{array}$ & $\begin{array}{l}1.105(0.22) \\
\text { CDE }\end{array}$ & $\begin{array}{l}1.344(0.83) \\
\mathrm{A}\end{array}$ & $\begin{array}{l}1.175(0.38) \\
\mathrm{B}\end{array}$ & $1.239 \mathrm{~A}$ \\
\hline YD & $\begin{array}{l}1.329(1.06) \\
\text { CDEF }\end{array}$ & $\begin{array}{l}1.086(0.18) \\
\mathrm{AB}\end{array}$ & $\begin{array}{l}1.364(0.95) \\
\mathrm{AB}\end{array}$ & $\begin{array}{l}1.187(0.43) \\
\mathrm{B}\end{array}$ & $\begin{array}{l}1.201(0.45) \\
\mathrm{B}\end{array}$ & $1.233 \mathrm{~A}$ \\
\hline $\mathrm{ZA}$ & $\begin{array}{l}1.346(0.83) \\
\text { BCDE }\end{array}$ & $\begin{array}{l}1.000(0.0) \\
B\end{array}$ & $\begin{array}{l}1.239(0.52) \\
A B C D\end{array}$ & $\begin{array}{l}1.076(0.18) \\
B C\end{array}$ & $\begin{array}{l}1.249(0.56) \\
\text { A }\end{array}$ & $1.182 \mathrm{~A}$ \\
\hline $\mathrm{ZB}$ & $\begin{array}{l}1.280(0.66) \\
\text { CDEF }\end{array}$ & $\begin{array}{l}1.077(0.16) \\
\mathrm{AB}\end{array}$ & $\begin{array}{l}1.232(0.52) \\
A B C D\end{array}$ & $\begin{array}{l}1.035(0.08) \\
\mathrm{BC}\end{array}$ & $\begin{array}{ll}1.262 & (0.59) \\
\mathrm{A} & \end{array}$ & $1.177 \quad \mathrm{~A}$ \\
\hline $\mathrm{ZC}$ & $\begin{array}{l}1.183(0.41) \\
\text { DEF }\end{array}$ & $\begin{array}{l}1.077(0.16) \\
\mathrm{AB}\end{array}$ & $\begin{array}{l}1.439(1.10) \\
\mathrm{A}\end{array}$ & $\begin{array}{l}1.070(0.15) \\
\mathrm{BC}\end{array}$ & $\begin{array}{l}1.287(0.66) \\
\mathrm{A}\end{array}$ & $1.211 \mathrm{~A}$ \\
\hline $\mathrm{ZD}$ & $\begin{array}{l}1.132(0.33) \\
\text { DEF }\end{array}$ & $\begin{array}{l}1.110(0.24) \\
\mathrm{AB}\end{array}$ & $\begin{array}{l}1.365(0.90) \\
\mathrm{AB}\end{array}$ & $\begin{array}{l}1.070(0.15) \\
B C\end{array}$ & $\begin{array}{l}1.257(0.58) \\
\mathrm{A}\end{array}$ & $1.187 \mathrm{~A}$ \\
\hline $\bar{U}$ & $1.697(1.88) \mathrm{A}$ & $\begin{array}{l}1.077(0.16) \\
\mathrm{AB}\end{array}$ & $\begin{array}{l}1.274(0.62) \\
\mathrm{ABC}\end{array}$ & $\begin{array}{l}1.083(0.18) \\
\text { BC }\end{array}$ & $\begin{array}{l}1.108(0.23) \\
\mathrm{C}\end{array}$ & $1.248 \quad \mathrm{~A}$ \\
\hline $\mathrm{T}$ & $\begin{array}{l}1.000(0.0) \\
\mathrm{F}\end{array}$ & $\begin{array}{l}1.000(0.0) \\
\mathrm{B}\end{array}$ & $\begin{array}{l}1.000(0.0) \\
\mathrm{E}\end{array}$ & $\begin{array}{l}1.000(0.0) \\
\mathrm{C}\end{array}$ & $\begin{array}{ll}1.000 & (0.00) \\
\mathrm{D} & \\
\end{array}$ & $1.000 \mathrm{~A}$ \\
\hline S.E. & \pm 0.08 & \pm 0.0581 & \pm 0.0697 & \pm 0.0555 & \pm 0.01581 & \pm 0.1020 \\
\hline C.V. $(\%)$ & 12.28 & 10.73 & 11.92 & 10.00 & 2.92 & \\
\hline
\end{tabular}

Note: Means followed by the same letter $(\mathrm{s})$ are not significantly different $(\mathrm{P}>0.05)$, according to D.M.R.T. Data transformed to $\sqrt{ } \mathrm{x}+1$. Actual means in parenthesis.

Legend: 1= Season 2001/2002, $2=$ Season 2002/2003, 3= Season 2003/2004, 4=Season 2004/2005 \& 5 =Season 2012/2013.

XA: 10 eggs and/or larvae/100 plants at pre-flowering stage, XB: 15 eggs and/or larvae/100 plants at pre-flowering stage,

$\mathrm{XC}$ : 20 eggs and/or larvae/100 plants at pre-flowering stage, $\quad \mathrm{XD}: 30$ eggs or 10 larvae/100 plants at pre-flowering stage,

YA: 10 eggs and/or larvae/100 plants at flowering stage, plants at flowering stage,

YC: 20 eggs and/or larvae/100 plants at flowering stage, plants at flowering stage,

ZA: 10 eggs and/or larvae/100 plants at boll formation stage, plants at boll formation stage,

ZC: 20 eggs and/or larvae/100 plants at boll formation stage, plants at boll formation stage, $\mathrm{U}$ : Infested throughout (untreated control), (Control).

YB: 15 eggs and/or larvae/100

YD: 30 eggs or 10 larvae/100

ZB: 15 eggs and/or larvae/100

ZD: 30 eggs or 10 larvae/100

$\mathrm{T}$ : Helicoverpa free infestation 
Table 3: Damaged cotton squares, flowers and bolls by $H$. armigera at different stages of growth, seasons; 2001/2002, 2002/2003, 2003/2004, 2004/2005 and 2012/2013. (Legends are same as in Table 2.)

\begin{tabular}{|c|c|c|c|c|c|c|c|c|c|c|c|c|c|c|c|c|c|c|c|c|c|c|c|c|c|}
\hline $\begin{array}{c}\text { Tr } \\
\text { ea } \\
\text { t. }\end{array}$ & \multicolumn{5}{|c|}{$\begin{array}{c}\text { \% damaged } \\
\text { squares }\end{array}$} & \multicolumn{5}{|c|}{$\begin{array}{c}\% \text { damaged } \\
\text { flowers }\end{array}$} & \multicolumn{5}{|c|}{$\begin{array}{c}\% \text { damaged } \\
\text { bolls }\end{array}$} & \multicolumn{5}{|c|}{$\begin{array}{l}\text { Mean No. of } \\
\text { eggs per } 100 \\
\text { plants }\end{array}$} & \multicolumn{5}{|c|}{$\begin{array}{c}\text { Mean No. of } \\
\text { larvae per } 100 \\
\text { plants }\end{array}$} \\
\hline & 1 & 2 & 3 & 4 & 5 & 1 & 2 & 3 & 4 & 5 & 1 & 2 & 3 & 4 & 5 & 1 & 2 & 3 & 4 & 5 & 1 & 2 & 3 & 4 & 5 \\
\hline $\begin{array}{l}X \\
A\end{array}$ & \begin{tabular}{|l|}
3 \\
0 \\
0
\end{tabular} & $\begin{array}{l}2 . \\
0\end{array}$ & $\begin{array}{l}6 . \\
9\end{array}$ & $\begin{array}{l}0 \\
4 \\
4\end{array}$ & $\begin{array}{l}16 \\
.9 \\
7\end{array}$ & & & & & & & & & & & \begin{tabular}{|l|}
5. \\
0
\end{tabular} & $\begin{array}{l}1 \\
0 \\
0\end{array}$ & $\begin{array}{l}4 \\
9 . \\
5\end{array}$ & \begin{tabular}{|l|}
0 \\
. \\
0
\end{tabular} & $\begin{array}{l}5 \\
3 \\
\end{array}$ & $\begin{array}{l}8 . \\
4\end{array}$ & \begin{tabular}{l|}
3 \\
0 \\
0
\end{tabular} & \begin{tabular}{l|}
1 \\
2. \\
0
\end{tabular} & $\begin{array}{l}0 \\
0 \\
0\end{array}$ & $\begin{array}{l}1 \\
1 . \\
3\end{array}$ \\
\hline $\begin{array}{l}\text { X } \\
B\end{array}$ & \begin{tabular}{|l|}
4 \\
0 \\
\end{tabular} & $\begin{array}{l}2 . \\
2\end{array}$ & $\begin{array}{l}8 . \\
5\end{array}$ & $\begin{array}{l}0 \\
4 \\
\end{array}$ & $\begin{array}{l}16 \\
.8 \\
9 \\
\end{array}$ & & & & & & & & & & & \begin{tabular}{|l|}
3 \\
5. \\
0 \\
\end{tabular} & \begin{tabular}{|l|}
0 \\
0 \\
\end{tabular} & $\begin{array}{l}3 \\
7 . \\
0 \\
\end{array}$ & \begin{tabular}{|l|}
0 \\
5 \\
\end{tabular} & $\begin{array}{l}5 \\
8 \\
\end{array}$ & $\begin{array}{l}6 . \\
3\end{array}$ & \begin{tabular}{l|}
3 \\
$\dot{0}$ \\
\end{tabular} & \begin{tabular}{l|}
1 \\
2. \\
5
\end{tabular} & $\begin{array}{l}0 \\
\dot{0} \\
\end{array}$ & $\begin{array}{l}1 \\
1 . \\
8\end{array}$ \\
\hline $\begin{array}{l}X \\
C\end{array}$ & \begin{tabular}{|l|}
5 \\
. \\
0 \\
\end{tabular} & $\begin{array}{l}1 . \\
9\end{array}$ & $\begin{array}{l}8 . \\
6\end{array}$ & $\begin{array}{l}0 \\
3 \\
\end{array}$ & \begin{tabular}{|l}
17 \\
.8
\end{tabular} & & & & & & & & & & & \begin{tabular}{|l|}
1 \\
7. \\
5 \\
\end{tabular} & \begin{tabular}{|l|}
0 \\
0 \\
\end{tabular} & $\begin{array}{l}5 \\
3 . \\
0 \\
\end{array}$ & \begin{tabular}{|l|}
0 \\
0 \\
\end{tabular} & $\begin{array}{l}6 \\
1 \\
\end{array}$ & $\begin{array}{l}1 \\
5 . \\
6 \\
\end{array}$ & \begin{tabular}{l|}
3 \\
$\dot{0}$ \\
\end{tabular} & \begin{tabular}{l|}
1 \\
4. \\
5
\end{tabular} & $\begin{array}{l}1 \\
\dot{0} \\
\end{array}$ & $\begin{array}{l}1 \\
1 . \\
9\end{array}$ \\
\hline $\begin{array}{l}X \\
D\end{array}$ & \begin{tabular}{|l|}
3 \\
9 \\
9 \\
\end{tabular} & \begin{tabular}{|l}
2. \\
5
\end{tabular} & $\begin{array}{l}1 \\
1 . \\
5\end{array}$ & $\begin{array}{l}0 \\
5 \\
\end{array}$ & \begin{tabular}{|l}
17 \\
.0 \\
4 \\
\end{tabular} & & & & & & & & & & & \begin{tabular}{|l|}
1 \\
6 \\
5 \\
\end{tabular} & \begin{tabular}{|l|}
0 \\
5 \\
\end{tabular} & \begin{tabular}{|l}
4 \\
5. \\
0 \\
\end{tabular} & \begin{tabular}{|l|}
0 \\
0 \\
\end{tabular} & \begin{tabular}{|l}
5 \\
8 \\
\end{tabular} & $\begin{array}{l}1 \\
2 . \\
5 \\
\end{array}$ & $\begin{array}{l}4 \\
5 \\
\end{array}$ & $\begin{array}{l}1 \\
9 . \\
0\end{array}$ & $\begin{array}{l}0 \\
. \\
0\end{array}$ & \begin{tabular}{|l}
1 \\
1. \\
8
\end{tabular} \\
\hline $\mathrm{U}$ & \begin{tabular}{|l|}
7 \\
0 \\
1 \\
\end{tabular} & \begin{tabular}{|l}
1 \\
6. \\
4
\end{tabular} & $\begin{array}{l}1 \\
6 . \\
8\end{array}$ & $\begin{array}{l}0 \\
6 \\
\end{array}$ & $\begin{array}{l}19 \\
.5\end{array}$ & & & & & & & & & & & \begin{tabular}{|l|}
7 \\
5. \\
0
\end{tabular} & \begin{tabular}{|l|}
0 \\
5 \\
\end{tabular} & \begin{tabular}{|l}
3 \\
8 \\
5 \\
\end{tabular} & \begin{tabular}{|l|}
1 \\
0 \\
0
\end{tabular} & \begin{tabular}{|l}
9 \\
2 \\
\end{tabular} & $\begin{array}{l}3 \\
7 . \\
5\end{array}$ & $\begin{array}{l}9 \\
5 \\
5\end{array}$ & $\begin{array}{l}2 \\
3 . \\
0\end{array}$ & $\begin{array}{l}0 \\
5 \\
5\end{array}$ & \begin{tabular}{|l}
1 \\
1. \\
4
\end{tabular} \\
\hline $\mathrm{T}$ & \begin{tabular}{|l|}
0 \\
0 \\
\end{tabular} & \begin{tabular}{|l|}
0. \\
0
\end{tabular} & $\begin{array}{l}0 . \\
0\end{array}$ & $\begin{array}{l}0 \\
0 \\
\end{array}$ & $\begin{array}{l}0 . \\
0\end{array}$ & & & & & & & & & & & \begin{tabular}{|l|}
0. \\
0
\end{tabular} & \begin{tabular}{|l|}
0 \\
0 \\
\end{tabular} & $\begin{array}{l}0 . \\
0\end{array}$ & \begin{tabular}{|l}
0 \\
0 \\
\end{tabular} & $\begin{array}{l}0 \\
0 \\
\end{array}$ & $\begin{array}{l}0 . \\
0\end{array}$ & $\begin{array}{l}0 \\
0 \\
0\end{array}$ & $\begin{array}{l}0 . \\
0\end{array}$ & $\begin{array}{l}0 \\
0 \\
0\end{array}$ & $\begin{array}{l}0 . \\
0\end{array}$ \\
\hline $\begin{array}{l}\mathrm{Y} \\
\mathrm{A}\end{array}$ & & & & & & \begin{tabular}{|l}
1 \\
0. \\
\end{tabular} & $\begin{array}{l}1 \\
2 \\
\end{array}$ & $\begin{array}{l}1 \\
0 . \\
3\end{array}$ & $\begin{array}{l} \\
2 \\
\end{array}$ & \begin{tabular}{|l}
3 \\
0. \\
1 \\
\end{tabular} & & & & & & $\begin{array}{l}3 . \\
1\end{array}$ & \begin{tabular}{|l|}
1 \\
5 \\
\end{tabular} & $\begin{array}{l}4 . \\
0\end{array}$ & \begin{tabular}{|l|}
0 \\
0 \\
\end{tabular} & $\begin{array}{l}8 \\
8 \\
7\end{array}$ & $\begin{array}{l}2 \\
8 . \\
1\end{array}$ & $\begin{array}{l}0 \\
0 \\
0\end{array}$ & $\begin{array}{l}2 \\
0 . \\
5\end{array}$ & \begin{tabular}{|l|}
1 \\
0 \\
0
\end{tabular} & $\begin{array}{l}4 . \\
3\end{array}$ \\
\hline $\begin{array}{l}\text { Y } \\
\text { B }\end{array}$ & & & & & & $\begin{array}{l}6 . \\
4\end{array}$ & 0 & $\begin{array}{l}9 . \\
4\end{array}$ & $\begin{array}{l}2 \\
0 \\
0\end{array}$ & $\begin{array}{l}3 \\
2 . \\
6\end{array}$ & & & & & & $\begin{array}{l}0 . \\
0\end{array}$ & \begin{tabular}{|l|}
0 \\
0 \\
0 \\
\end{tabular} & $\begin{array}{l}6 . \\
5\end{array}$ & \begin{tabular}{|l|}
0 \\
0 \\
0
\end{tabular} & \begin{tabular}{|l}
8 \\
4 \\
\end{tabular} & $\begin{array}{l}6 . \\
3\end{array}$ & $\begin{array}{l}0 \\
\dot{0} \\
\end{array}$ & $\begin{array}{l}1 \\
6 . \\
0\end{array}$ & $\begin{array}{l}0 \\
5 \\
5\end{array}$ & $\begin{array}{l}5 . \\
3\end{array}$ \\
\hline $\begin{array}{l}\mathrm{Y} \\
\mathrm{C}\end{array}$ & & & & & & $\begin{array}{l}1 \\
0 . \\
9\end{array}$ & 1 & \begin{tabular}{l|}
1 \\
0. \\
0
\end{tabular} & $\begin{array}{l}1 \\
\dot{0}\end{array}$ & $\begin{array}{l}3 \\
1 . \\
4\end{array}$ & & & & & & $\begin{array}{l}9 . \\
4\end{array}$ & \begin{tabular}{l|}
0 \\
0 \\
0
\end{tabular} & $\begin{array}{l}9 . \\
5\end{array}$ & \begin{tabular}{|l|}
0 \\
5 \\
5
\end{tabular} & $\begin{array}{l}8 \\
8 \\
6\end{array}$ & $\begin{array}{l}1 \\
5 . \\
6\end{array}$ & $\begin{array}{l}0 \\
\dot{0}\end{array}$ & $\begin{array}{l}1 \\
6 . \\
0\end{array}$ & \begin{tabular}{l|}
1 \\
0 \\
0
\end{tabular} & $\begin{array}{l}5 . \\
7\end{array}$ \\
\hline $\begin{array}{l}\mathrm{Y} \\
\mathrm{D}\end{array}$ & & & & & & $\begin{array}{l}8 . \\
0\end{array}$ & \begin{tabular}{|l}
5 \\
5 \\
\end{tabular} & $\begin{array}{l}1 \\
1 \\
1 \\
\end{array}$ & \begin{tabular}{|l} 
\\
2 \\
\end{tabular} & $\begin{array}{l}2 \\
5 . \\
6 \\
\end{array}$ & & & & & & $\begin{array}{l}6 . \\
3\end{array}$ & \begin{tabular}{|l|}
0 \\
0 \\
0 \\
\end{tabular} & $\begin{array}{l}5 . \\
0\end{array}$ & \begin{tabular}{|l|}
1 \\
0 \\
0
\end{tabular} & \begin{tabular}{|l|}
6 \\
3 \\
\end{tabular} & $\begin{array}{l}1 \\
2 . \\
5\end{array}$ & $\begin{array}{l}0 \\
0 \\
0\end{array}$ & $\begin{array}{l}3 \\
6 . \\
5\end{array}$ & \begin{tabular}{|l|}
1 \\
0 \\
\end{tabular} & $\begin{array}{l}6 . \\
3\end{array}$ \\
\hline $\mathrm{U}$ & & & & & & \begin{tabular}{|l}
2 \\
7. \\
1
\end{tabular} & 1 & $\begin{array}{l}9 . \\
5\end{array}$ & \begin{tabular}{|l|}
2 \\
3 \\
\end{tabular} & $\begin{array}{l}2 \\
2 . \\
9\end{array}$ & & & & & & $\begin{array}{l}2 \\
5 . \\
0\end{array}$ & \begin{tabular}{|l|}
0 \\
$\dot{0}$ \\
\end{tabular} & $\begin{array}{l}8 . \\
0\end{array}$ & \begin{tabular}{|l|}
0 \\
0 \\
0
\end{tabular} & \begin{tabular}{|l|}
3 \\
6 \\
\end{tabular} & $\begin{array}{l}4 \\
3 . \\
8\end{array}$ & $\begin{array}{l}0 \\
0 \\
0\end{array}$ & $\begin{array}{l}2 \\
1 . \\
5\end{array}$ & \begin{tabular}{|l|}
0 \\
\\
\end{tabular} & \begin{tabular}{|l}
3. \\
3
\end{tabular} \\
\hline $\mathrm{T}$ & & & & & & $\begin{array}{l}0 . \\
0\end{array}$ & 0 & $\begin{array}{l}0 . \\
0\end{array}$ & $\begin{array}{l}0 \\
0 \\
\end{array}$ & $\begin{array}{l}0 . \\
0\end{array}$ & & & & & & $\begin{array}{l}0 . \\
0\end{array}$ & \begin{tabular}{|l|}
0 \\
\\
0
\end{tabular} & $\begin{array}{l}0 . \\
0\end{array}$ & $\begin{array}{l}0 \\
0 \\
0\end{array}$ & $\begin{array}{l}0 \\
0 \\
0\end{array}$ & $\begin{array}{l}0 . \\
0\end{array}$ & $\begin{array}{l}0 \\
0 \\
0\end{array}$ & $\begin{array}{l}0 . \\
0\end{array}$ & \begin{tabular}{|l|}
0 \\
0 \\
\end{tabular} & $\begin{array}{l}0 . \\
0\end{array}$ \\
\hline $\begin{array}{l}\mathrm{Z} \\
\mathrm{A}\end{array}$ & & & & & & & & & & & $\begin{array}{l}1 \\
0 \\
0\end{array}$ & 0 & 1 & 0 & 6 & $\begin{array}{l}0 . \\
0\end{array}$ & \begin{tabular}{|l|}
0 \\
0 \\
0
\end{tabular} & $\begin{array}{l}0 . \\
0\end{array}$ & $\begin{array}{l}0 \\
0 \\
0\end{array}$ & $\begin{array}{l}0 \\
9 \\
9\end{array}$ & $\begin{array}{l}4 . \\
2\end{array}$ & $\begin{array}{l}0 \\
0 \\
0\end{array}$ & $\begin{array}{l}1 \\
7 . \\
0\end{array}$ & \begin{tabular}{|l|}
0 \\
5
\end{tabular} & $\begin{array}{l}5 . \\
6\end{array}$ \\
\hline $\begin{array}{l}\mathrm{Z} \\
\mathrm{B}\end{array}$ & & & & & & & & & & & 0 & 0 & 1 & 0 & \begin{tabular}{|l}
7 \\
. \\
4
\end{tabular} & $\begin{array}{l}8 . \\
3\end{array}$ & \begin{tabular}{|l|}
0 \\
0 \\
0 \\
\end{tabular} & $\begin{array}{l}0 . \\
0\end{array}$ & $\begin{array}{l}0 \\
0 \\
\end{array}$ & \begin{tabular}{|l|}
0 \\
6 \\
\end{tabular} & $\begin{array}{l}8 . \\
3\end{array}$ & $\begin{array}{l}0 \\
0 \\
0\end{array}$ & $\begin{array}{l}1 \\
4 . \\
0\end{array}$ & \begin{tabular}{|l|}
0 \\
0 \\
\end{tabular} & $\begin{array}{l}7 . \\
1\end{array}$ \\
\hline
\end{tabular}




\begin{tabular}{|l|l|l|l|l|l|l|l|l|l|l|l|l|l|l|l|l|l|l|l|l|l|l|l|l|}
\hline $\mathrm{Z}$ \\
$\mathrm{C}$
\end{tabular}

Different chemical applications were carried out with selective pesticides (Table 4). Avaunt 105 SC at 0.105 litre/fed was used in the first four seasons and the insecticide Tracer 240 SC (spinosad) at 0.104 litre/fed was used in the last season. Seasons 2001/2002, 2003/2004 and $2012 / 2013$ were subjected to a number of chemical sprays due to the high level of infestation by H. armigera, especially in season 2003/2004. It is cleared that in an implementation of an IPM program in agricultural crops the use of economic thresholds is an important element, if a chemical application is executed only when it is needed, a reduction in labour and pesticide costs may be achieved and the advantages on the long-term are the build-up of beneficial organisms (natural enemies) (Barendregt and van Lenteren, 1992). Moreover, the probability of insecticide resistance in the target pest decreases and reduction in chemical spraying has positive impacts on the environment (Munrow, 1987).

Table 4: The number of chemical sprays, according to the suggested action threshold levels, Seasons; 2001/2002, 2002/2003, 2003/2004, 2004/2005 and 2012/2013. (Legends are same as in Table 2.)

\begin{tabular}{|c|c|c|c|c|c|}
\hline Treatments & \multicolumn{6}{|c|}{ Number of sprays } \\
\hline & $2001 / 2002$ & $2002 / 2003$ & $2003 / 2004$ & $2004 / 2005$ & $2012 / 2013$ \\
\hline XA & 1 & 1 & 5 & 0 & 4 \\
\hline XB & 2 & 0 & 4 & 0 & 3 \\
\hline XC & 1 & 0 & 3 & 0 & 2 \\
\hline XD & 1 & 1 & 3 & 0 & 3 \\
\hline YA & 1 & 0 & 4 & 0 & 2 \\
\hline YB & 0 & 0 & 3 & 0 & 2 \\
\hline YC & 0 & 0 & 3 & 0 & 1 \\
\hline YD & 0 & 0 & 2 & 0 & 1 \\
\hline ZA & 0 & 0 & 2 & 0 & 1 \\
\hline ZB & 0 & 0 & 2 & 0 & 0 \\
\hline ZC & 0 & 0 & 1 & 0 & 0 \\
\hline ZD & 0 & 0 & 2 & 0 & 0 \\
\hline U & 0 & 0 & 0 & 0 & 0 \\
\hline T & 0 & 0 & 0 & 0 & 0 \\
\hline
\end{tabular}

The data presented on seed cotton yield showed that there were no significant difference between the levels $\mathrm{A}, \mathrm{B}, \mathrm{C}$ and $\mathrm{D}$ within the three stages of cotton growth $\{(\mathrm{P}>0.05)$, according to 
DMRT (Table 5). Apparently, increasing number of sprays did not result in economically justifiable increase in yield. Therefore, it is cleared that on the advanced flowering stage, chemical interference could be started with reference to the action threshold D (30 eggs or 10 larvae/ 100 plants). This mean that the findings of this study to some extent come with the findings of the IPM Project released in 1993 which could reduce the number of chemical sprays and offers better chance to the natural enemies to play a role in pest suppression, especially in the early season, in addition to the other positive impacts.

Table 5: Mean seed cotton yield (k.p.f.), seasons; 2001/2002, 2002/2003, 2003/2004, 2004/2005 and 2012/2013. (Legends are same as in Table 2.)

\begin{tabular}{|c|c|c|c|c|c|c|}
\hline Treatment & & \multicolumn{4}{|c|}{ Yield (k.p.f.) } & Combined \\
\hline & $2001 / 2002$ & $2002 / 2003$ & $2003 / 2004$ & $2004 / 2005$ & $2012 / 2013$ & \\
\hline $\mathrm{XA}$ & $5.49 \mathrm{AB}$ & 3.95 & $5.35 \mathrm{ABCD}$ & 1.71 & 6.73 & $4.646 \mathrm{ABC}$ \\
\hline $\mathrm{XB}$ & $5.85 \mathrm{~A}$ & 3.87 & $5.65 \mathrm{ABC}$ & 1.89 & 6.71 & $4.794 \mathrm{AB}$ \\
\hline $\mathrm{XC}$ & $5.20 \mathrm{AB}$ & 3.78 & $5.86 \mathrm{ABC}$ & 1.90 & 6.64 & $4.676 \mathrm{ABC}$ \\
\hline $\mathrm{XD}$ & $4.45 \quad \mathrm{~B}$ & 3.84 & $5.23 \mathrm{ABCDE}$ & 1.92 & 6.81 & $4.450 \mathrm{ABC}$ \\
\hline YA & $5.17 \mathrm{AB}$ & 3.78 & $6.28 \mathrm{~A}$ & 2.26 & 6.25 & $4.748 \mathrm{AB}$ \\
\hline YB & $5.48 \mathrm{AB}$ & 3.92 & $5.89 \mathrm{ABC}$ & 2.22 & 7.05 & $4.912 \mathrm{~A}$ \\
\hline $\mathrm{YC}$ & $5.28 \mathrm{AB}$ & 3.62 & $6.11 \mathrm{AB}$ & 2.11 & 7.18 & $4.860 \mathrm{~A}$ \\
\hline YD & $4.79 \mathrm{AB}$ & 3.76 & $\mathrm{CDE}$ & 1.83 & 6.01 & $4.260 \quad \mathrm{BC}$ \\
\hline $\mathrm{ZA}$ & $5.16 \mathrm{AB}$ & 4.40 & $5.10 \quad \mathrm{BCDE}$ & 1.97 & 6.32 & $4.590 \mathrm{ABC}$ \\
\hline $\mathrm{ZB}$ & $4.69 \mathrm{AB}$ & 4.16 & $5.28 \mathrm{ABCD}$ & 2.04 & 6.3 & $4.494 \mathrm{ABC}$ \\
\hline $\mathrm{ZC}$ & $5.36 \mathrm{AB}$ & 4.27 & 4.36 & 2.20 & 6.14 & $4.466 \mathrm{ABC}$ \\
\hline ZD & $4.76 \mathrm{AB}$ & 4.07 & $\mathrm{CDE}$ & 1.93 & 5.83 & $4.286 \quad \mathrm{BC}$ \\
\hline $\mathrm{U}$ & $4.72 \mathrm{AB}$ & 3.80 & 4.16 & 2.01 & 6.16 & $4.170 \quad \mathrm{C}$ \\
\hline $\mathrm{T}$ & $4.93 \mathrm{AB}$ & 4.02 & 4.45 & 2.11 & 6.43 & $4.388 \mathrm{ABC}$ \\
\hline S.E. \pm & 0.87 & 1.2457 & 1.300 & 0.1999 & 1.865 & 0.1655 \\
\hline C.V. (\%) & 15.95 & 15.78 & 12.39 & 19.92 & $13.45 \%$ & \\
\hline
\end{tabular}

From the yields and number of sprays in the treatments it can be concluded that the already recommended action threshold 30 eggs or 10 larvae/100 plants is still be applied when flowering advances and bolls formed. Moreover, the standing action threshold seems lower than the actual level. Therefore, it is suggested to investigate higher action threshold than that in current use.

\section{Conclusion}

It is concluded that the action threshold level for the African bollworm, Helicoverpa armigera (Hbn.) on cotton is 30 eggs or 10 larvae/100 plants to be applied when flowering advances and bolls formed until a new action threshold level established.

\section{Acknowledgements}

The authors are grateful to the administration of the Integrated Agricultural Pests Management Research Centre for supporting this study. Grateful also extended to the technical staff of the IPM Research and Training Department together with the staff of Prof. Elamin M. Elamin, Mr. Mohamed E. El Khawad and Dr. Sara Abdelraheem. Special thanks to Dr. Ahmed Hassan 
Mohamed for reviewing the last version of the manuscript. Also thanks extended to Prof. Hassan O. Kennan and Prof. Tag Elsir Elamin Abdalla for their technical support.

\section{References}

[1] Abbelrahman, A. A., Stam, P. A. and Munir B. (1991). Recommendation for increased economic threshold levels for Heliothis armigera, Empoasca lybica, Aphis gosspyii and Bemisia tabaci on cotton in Sudan. Wad Medani, 57th National Pests and Diseases Committee Meeting, 3 June, 1991, 48 pp.

[2] Abbelrahman, A. A. (1995). Delaying chemical control for Helicoverpa armigera (Hb.) in cotton in Sudan to promote IPM. Crop Protection.

[3] Balla, A. N. (1979). American bollworm in the Gezira and Managil. In: Siddig, M. A. and Hughes, L. C. (eds.) Cotton growth in the Gezira environment. Agricultural Research Corporation, Sudan. Wad Medani, p. 281-291.

[4] Barendregt, H. E. and van Lenteren, J. C. (1992). Aspects of economic thresholds of insect pests in cotton: an annotated bibiliography. FAO/GCP/SUD/025/NET, Sudan and Department of Entomology, Agricultural Universty Wageningen. pp. 100.

[5] Eveleens, K. G. and Abbelrahman, A. A. (1980). The cotton whitefly problem: can the tide be turned? Working paper No. 5; FAO/UNEP African Programme for the development and Application of integrated Pest Control in Cotton Growing prepared for the 44th Meeting of the National Pests and Diseases Committee, ARC Gezira Research Station, Mimeograph 12 pp.

[6] Munrow, J. M. (1987). Cotton. Longman Scientific and Technical, Harlow.

[7] Schmutterer, H. (1969). Pests of Crops in Northeast and Central Africa. Gustav Fischer, Verlag, Stuttgart. Portland USA. pp 178-182.

[8] Stam, P. A., Abdelrahman, A.A. and Munir, B. (1994). Comparisons of control action threshold for Heliothis armigera, Bemisia tabaci and Aphis gossypii on cotton in the Sudan Gezira and Rahad regions. Crop. Protec. 13:503-512.

[9] Walker, P. T. (1971). In ed. L. Chiarapa, Crop Loss Assessment Methods, FAO Manual on the evaluation and prevention of losses by pests, disease and weeds. Alden and Mowbray Ltd. at the Alden Press, Oxford. $112 \mathrm{p}$.

[10] Wallach, D. (1980). An empirical mathematical model of a cotton crop subjected to damage. Field crops research 3: 7-25.

\footnotetext{
*Corresponding author.

E-mail address: aaymalik@ yahoo.com
} 